\title{
Photodynamic diagnosis model for depth evaluation of basal cell carcinomas
}

\author{
I. Salas-García, F. Fanjul-Vélez, N. Ortega-Quijano, J. L. Arce-Diego \\ Applied Optical Techniques Group, TEISA Department, University of Cantabria, Av. de los Castros S/N, 39005 Santander, Spain \\ isalas@teisa.unican.es,fanjulf@unican.es, ortegan@unican.es, arcedj@unican.es
}

\begin{abstract}
The present work is devoted to the development of a diagnostic model based on photodynamic therapy to obtain an estimation of a skin tumor depth from the measurement of the photosensitizer fluorescence emission.

OCIS codes: (170.5180) Photodynamic therapy; (170.4580) Optical diagnostics for medicine; (260.2510) Fluorescence; (260.5130) Photochemistry
\end{abstract}

\section{Introduction}

Photodynamic Therapy (PDT) [1] is commonly known for its excellent therapeutic properties to treat non melanoma skin cancers and cancer precursor diseases although its diagnostic capability is fairly unexploited. The photosensitizer fluorescence diagnosis or photodynamic diagnosis allows a macroscopic evaluation and the delimitation of the tumor superficial margins [2]. However a microscopic evaluation in depth requires the use of other techniques such as confocal and tomographic techniques. The present work is devoted to the application of a PDT based diagnostic model to obtain an estimation of a skin tumor depth from the measurement of the photosensitizer fluorescence emission [3]. The obtained results so far allow us to know the photosensitizer fluorescence emission during the PDT treatment and constitute a first approach to achieve a future model that permits to diagnose the extent in depth of a tumor using superficial photosensitizer fluorescence measurements.

\section{Photodynamic diagnostic modeling}

The PDT based diagnostic model takes into account the optical radiation distribution, an inhomogeneous topical photosensitizer distribution in the target tissue, the photochemical interaction that takes place between the optical radiation and the photosensitized tissue and the photosensitizer fluorescence emission. In order to obtain the distribution of light in a three dimensional tissue we used the Radiation Transport Theory (RTT) [4] in a steady-state situation without sources inside the tissue:

$$
\hat{\mathrm{s}} \cdot \bar{\nabla} \mathrm{I}(\mathrm{r}, \hat{\mathrm{s}})=-\left(\mu_{\mathrm{a}}+\mu_{\mathrm{s}}\right) \mathrm{I}(\mathrm{r}, \hat{\mathrm{s}})+\frac{\mu_{\mathrm{s}}}{4 \pi} \int_{4 \pi} \mathrm{p}\left(\hat{\mathrm{s}}^{\cdot \hat{\mathrm{s}}^{\prime}}\right) \mathrm{I}\left(\mathrm{r}, \hat{\mathrm{s}}^{\prime}\right) \mathrm{d} \Omega^{\prime}
$$

The excitation optical radiation distribution was obtained by means of a Monte Carlo approach [5] while the photosensitizer fluorescence emission generated was obtained taking into account the spatial temporal variation of photosensitizer molecules during the photochemical interaction, the excitation photon density and the fluorescence quantum yield. Thus the photosensitizer fluorescence power density generated was calculated as:

$$
\mathrm{P}_{\mathrm{f}}=\mathrm{v} \cdot \rho \cdot \sigma_{\mathrm{psa}} \cdot\left[\mathrm{S}_{0}\right] \cdot \eta_{10} \cdot \mathrm{E}_{\text {photon }_{\lambda \mathrm{em}}}
$$

where $\mathrm{E}_{\mathrm{photon}_{\lambda \mathrm{em}}}=\mathrm{h} \cdot \mathrm{c} / \lambda_{\mathrm{em}}$ is the photon energy at the fluorescence emission wavelength, $\mathrm{h}$ is the Planck's constant, $\mathrm{c}$ is the speed of light in vacuum and $\lambda_{\mathrm{em}}$ is the fluorescence emission wavelength.

The final inhomogeneous photosensitizer distribution in the target tissue during the incubation period was obtained by means of the Fick's law shown in equation (3), where $\mathrm{J}$ is the flux vector indicating the direction and magnitude of substance, $\mathrm{D}$ is the diffusion coefficient, $\mathrm{M}$ is the prodrug concentration and $\mathrm{z}$ is the depth in the tissue.

$$
J=-D \frac{\partial M}{\partial z}
$$


The temporal evolution of the photosensitizer precursor concentration, $M$, for each depth in the tissue sample can be calculated as, [6]:

$$
M(t)=M_{o} \int_{0}^{t}\left(\frac{K}{\sqrt{D \pi t^{\prime}}} e^{-\frac{z^{2}}{4 D t^{\prime}}}-\frac{K^{2}}{D} e^{\frac{K}{D} z} e^{\frac{K^{2}}{D} t^{\prime}} \operatorname{erfc}\left(\frac{K}{\sqrt{D}} \sqrt{t^{\prime}}+\frac{z}{2 \sqrt{D t^{\prime}}}\right)\right) e^{-\frac{t^{\prime}}{\tau}} d t^{\prime}
$$

where $M_{o}$ is the concentration of the photosensitizer precursor in the skin surface at $t=0, z$ is the distance from the corneal layer located at $\mathrm{z}=0, \mathrm{~K}$ is the permeability of the stratum corneum and $\tau$ is the relaxation time of the precursor as a consequence of the generation of the photosensitizer and other processes (lymphatic flow and blood perfusion). The concentration of photoactive substance, $\mathrm{S}_{0}$, accumulated in the tissue was obtained as expressed in (5), assuming that the photosensitizer relaxation time is fast compared to the photosensitizer precursor diffusion time, $\tau_{\mathrm{p}} \ll \mathrm{t}$. Therefore the concentration of photoactive compound, [6], is proportional to the instantaneous value of the precursor concentration, $\varepsilon_{p}$ is the yield of the conversion process and $\tau_{a \rightarrow p}$ the relaxation time of the photosensitizer precursor due to the generation of the photoactive compound.

$$
S_{0}(t)=\varepsilon_{p} \frac{\tau_{p}}{\tau_{a \rightarrow p}} M(t)
$$

The temporal evolution of the photosensitizer molecules and other molecular components involved in the photochemical interaction between the optical radiation and the photosensitized tissue was obtained by means of a photochemical model [7,8]. This model is based on a stiff differential equations system (equations 6 to 11) which takes into account the energetic transitions of the photosensitizer during a Type II reaction. The solutions of the stiff differential equations system employed were obtained by means of a differential equation solver within the Matlab ${ }^{\circledR}$ platform.

$$
\begin{gathered}
\frac{d\left[S_{0}\right]}{d t}=-v \rho \sigma_{p s a}\left[S_{0}\right]-k p b\left[{ }^{1} O_{2}\right]\left[S_{0}\right]+\frac{\eta_{10}}{\tau 1}\left[S_{1}\right]+\frac{\eta_{30}}{\tau 3}[T]+\frac{\alpha s}{\tau 3}[T]\left[{ }^{3} O_{2}\right](6) \\
\frac{d\left[S_{1}\right]}{d t}-\frac{1}{\tau 1}\left[S_{1}\right]+v \rho \sigma_{p s a}\left[S_{0}\right] \\
\frac{d[T]}{d t}=-\frac{\eta_{30}}{\tau 3}[T]-\frac{\alpha s}{\tau 3}[T]\left[{ }^{3} O_{2}\right]+\frac{\eta_{13}}{\tau 1}\left[S_{1}\right] \\
\frac{\left.d{ }^{3} O_{2}\right]}{d t}=-\frac{\alpha s}{\tau 3}[T]\left[{ }^{3} O_{2}\right]+\frac{\eta_{0}}{\tau 0}\left[{ }^{1} O_{2}\right]+P \\
\left.\frac{d\left[{ }^{1} O_{2}\right]}{d t}=-k p b\left[S_{0}\right]\left[{ }^{1} O_{2}\right]-k c x[R]\left[{ }^{1} O_{2}\right]-k s c[C]\right]_{i}\left[{ }^{1} O_{2}\right]-\frac{\eta_{0}}{\tau_{0}}\left[{ }^{1} O_{2}\right]+\frac{\alpha s}{\tau 3}[T]\left[{ }^{3} O_{2}\right] \\
\frac{d[R]}{d t}=-k c x\left[{ }^{1} O_{2}\right][R]+U
\end{gathered}
$$

\section{Results and discussion}

Figure 1 shows the fluorescence power density generated by the photosensitizer molecules at different temporal points during the photodynamic treatment. As it can be observed, the fluorescence decreases more rapidly in shallow 
areas where a greater amount of energy is deposited and the photobleaching effects appear first. As the optical irradiation is applied during the progression of treatment, most of the fluorescence comes from the inner layers of the tumor.
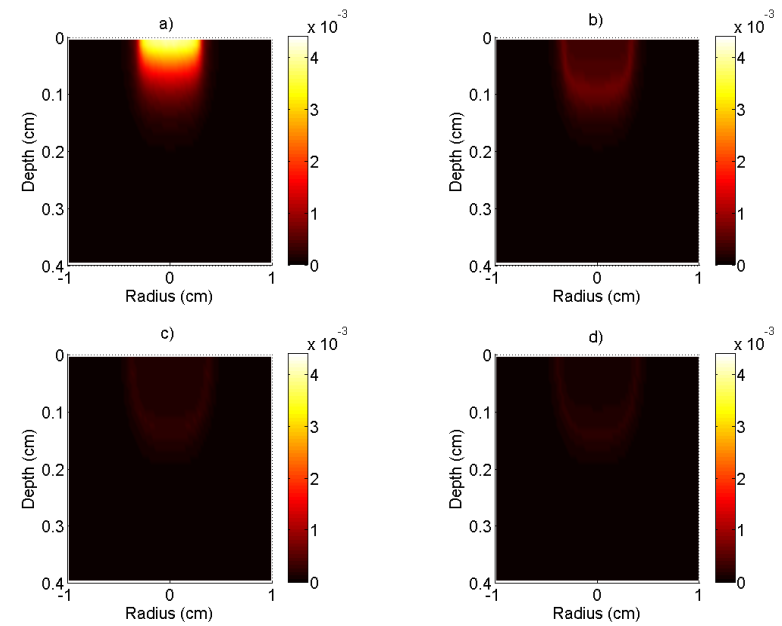

Fig. 1. Photosensitizer fluorescence power density $\left(\mathrm{W} / \mathrm{cm}^{3}\right)$ generated at a) $\left.\left.5 \mathrm{~s}, \mathrm{~b}\right) 50 \mathrm{~s}, \mathrm{c}\right) 120 \mathrm{~s}$ and d) $150 \mathrm{~s}$ during PDT

The results shown correspond to a basal cell carcinoma tumor depth of $3 \mathrm{~mm}$, irradiated with a cylindrical laser beam with a radius of $0.3 \mathrm{~cm}$ perpendicular to the tumor surface and a fixed irradiance of $100 \mathrm{~mW} / \mathrm{cm}^{2}$. The optical properties used at the excitation wavelength $(635 \mathrm{~nm})$ for the tumor were the absorption coefficient, $\mu_{\mathrm{a}}=1.5 \mathrm{~cm}^{-1}$, the scattering coefficient, $\mu_{\mathrm{s}}=104.76 \mathrm{~cm}^{-1}$, the anisotropy of scattering, $\mathrm{g}=0.79$ and the index of refraction, $\mathrm{n}=1.5$, whereas the healthy tissue beneath the tumor was considered muscle with an index of refraction of 1.37 . The photosensitizer precursor Methyl Aminolevulinate (MAL) was used to model its diffusion through the tumor tissue and to obtain the photosensitizer Protoporphyrin IX (PpIX) endogenously generated during an incubation period of 3 hours. In view of the results presented in Figure 1, the selective localization of the photoactive substance in the tumor and its ability to emit fluorescence coupled with the fact that the photosensitizer fluorescence comes mostly from the inner layers of the tumor as the excitation time increases, could be employed to get a relationship between the time period when fluorescence is emitted and the depth of the tumor. The model presented and these preliminary results will constitute the starting point of future works to develop a model based in the ability of the photosensitizer molecules to emit fluorescence applied in PDT with a diagnostic purpose instead of with a therapeutic one. The main objective of this diagnostic model based on PDT will be to estimate the depth of the tumor by means of the analysis of the time needed to get a significant decrease in the fluorescence.

\section{References}

[1] T. J. Dougherty, C. J. Gomer, B. W. Henderson, G. Jori, D. Kessel, M. Korbelik, J. Moan, and Q. Peng, "Photodynamic therapy” J. Natl. Cancer Inst. 90, 889-905 (1998)

[2] W. Bäumler, C. Abels and R. M. Szeimies, "Fluorescence Diagnosis and Photodynamic Therapy in Dermatology", Med. Laser Appl. 18, 47$56(2003)$.

[3] I. Salas-García, F. Fanjul-Vélez and J. L. Arce-Diego, "Spatial photosensitizer fluorescence emission predictive analysis for photodynamic therapy monitoring applied to a skin disease", Optics Communications, in press

[4] T. Vo-Dinh, Biomedical Photonics handbook, CRC Press, Boca Raton, 2003.

[5] L. Wang, S. L. Jacques and L. Zheng, "MCML - Monte Carlo modeling of light transport in multi-layered tissues" Computer methods and programs in biomedicine 47, 131-146 (1995).

[6] L.O. Svaasand, P. Wyss, M.T. Wyss, Y. Tadir, B.J. Tromberg and M.W. Berns, "Dosimetry Model for Photodynamic Therapy With Topically Administered Photosensitizers", Lasers in Surgery and Medicine 18, 139-149 (1996).

[7] T. H. Foster, R. S. Murant, R. G. Bryant, R. S. Knox, S. L. Gibson and R. Hilf, "Oxygen Consumption and Diffusion Effects in PDT", Radiation Research 126(3), 296-303 (1991).

[8] X. H. Hu, Y. Feng, J. Q. Lu, R. R. Allison, R. E. Cuenca, G. H. Downie and C. H. Sibata, "Modeling of a type II Photofrin-mediated PDT process in a heterogeneous tissue phantom", Photochemistry and Photobiology 81, 1460-1468 (2005). 